\title{
Are Hispanic patients with subarachnoid hemorrhage treated differently in border states than in nonborder states?
}

\author{
Ameer E. Hassan, DO, ${ }^{1,2}$ Gabriela A. Villanueva, MS, ${ }^{2}$ Ahmed A. Malik, MD, ${ }^{3}$ Sonia Shariff, BS, ${ }^{1}$ and \\ Adnan I. Qureshi, MD ${ }^{3}$ \\ 1Department of Neurology and Radiology, Valley Baptist Medical Center, University of Texas Health Science Center-San \\ Antonio, Harlingen, Texas; ${ }^{2}$ School of Medicine, University of Texas Health Science Center-San Antonio, Texas; and ${ }^{3 Z}$ Zeenat \\ Qureshi Stroke Institute, University of Minnesota, Minneapolis, Minnesota
}

\begin{abstract}
OBJECTIVE Recent studies have found an underutilization of in-hospital procedures in treatments of Hispanic patients admitted with coronary artery disease in states along the US-Mexico border ("border states"). The purpose of this study was to determine any treatment disparities between patients treated for subarachnoid hemorrhage (SAH) in border and nonborder states and whether this disparity was associated with differential hospital charges.
\end{abstract}

METHODS Using the National (Nationwide) Inpatient Sample, the authors retrieved data of Hispanic and non-Hispanic patients who were admitted in 2011 for SAH in a border state (California, Arizona, New Mexico, and Texas) or nonborder state (the remaining 46 US states). The authors determined the rates of use of endovascular coiling and surgical clipping treatments, hospital charges, and outcomes according to the patients' demographics and treatment in border or nonborder states.

RESULTS In total, 18,368 patients were admitted with SAH in the selected time period, including 2310 Hispanic patients (12.6\%). Of these patients, 1525 were admitted in a border state and 785 in a nonborder state. In border states, rates of surgical treatment significantly differed between patients of Hispanic (21.9\%) and non-Hispanic (14.0\%) origin $(p=0.02)$. In particular, Hispanic patients were more likely to undergo surgical clipping than were non-Hispanic patients. In the nonborder states, the rates of surgical treatment were similar for Hispanic and non-Hispanic patients (14.0\% vs $15.6 \%, p$ $=0.6$ ). Hispanic patients with SAH were billed significantly higher in-hospital charges in border states than in nonborder states (\$219,260 and $\$ 192,418$ [US dollars], respectively, $p<0.001)$.

CONCLUSIONS Use of surgical treatments for Hispanic patients with SAH residing in border states has a unique pattern, which significantly increases in-hospital charges in this patient population.

https://thejns.org/doi/abs/10.3171/2016.6.JNS152476

KEY WORDS border; endovascular; health disparities; Hispanic; subarachnoid hemorrhage; surgical; vascular disorders

$\mathrm{C}$ ONSTITUTING about $17 \%$ of the US population, Hispanics are the fastest growing minority group in the United States. This group also has the highest prevalence of cardiovascular risk factors, such as hypertension, diabetes mellitus, coronary artery disease, and physical inactivity.,13 It is projected that over the next 4 decades, 1.3 million strokes will occur each year in the United States, and Hispanics will contribute a large portion to this number. ${ }^{6}$ Subarachnoid hemorrhage (SAH), a specific type of stroke, is a major cause of disability and death and affects every 5 in 10,000 Hispanics. ${ }^{12}$ The rate of increase in this population and the expected increase of stroke incidence among Hispanics calls for innovative research that examines the current disparity gaps in prevention, prevalence, and treatment of SAH, which all result in detrimental outcomes for Hispanic patients.

Most studies have focused on the vast health care disparities between Hispanics and non-Hispanics; however, recent studies have also reported a growing disparity between Hispanics residing in border states and those living in nonborder states. Nearly three-quarters of Hispanic residents are situated in states along the US-Mexico border, 
that is, in Arizona, California, New Mexico, and Texas. ${ }^{3}$ Hispanics living in these states tend to have higher poverty rates, less access to medical care, and be less acculturated to US health care. ${ }^{2}$ For instance, a study by Gillum et al. reported significant health care disparities between Hispanics in border states and Hispanics in nonborder states receiving care for coronary artery disease. ${ }^{3}$ The authors observed an underutilization of hospital inpatient care for coronary artery disease in Hispanics living in the border states Texas, Arizona, and California compared with Hispanics living in nonborder states. Likewise, other studies have shown that Hispanics are less likely than non-Hispanic whites to be treated with recombinant tissue plasminogen activator for acute ischemic stroke. ${ }^{9}$ This observation poses the question whether other treatments, such as treatments for SAH (i.e., surgical clipping, coil embolization, or craniotomy), might also be underused in Hispanics living in border or nonborder states.

The purpose of the current study was to determine whether a disparity in SAH treatment and treatment costs exists between Hispanic and non-Hispanic patients in border and nonborder states. In addition, this study investigated whether Hispanic patients with SAH in border or nonborder states underwent different types of treatment and whether any such differences resulted in disparities in the cost of care.

\section{Methods}

For the analyses in the present study, we used data files from the National (Nationwide) Inpatient Sample (NIS), covering the year 2011. A comprehensive synopsis of the nature of the NIS data is available at http://www.hcup-us. ahrq.gov.

We used the International Classification of Disease, Ninth Revision, Clinical Modification (ICD-9-CM) primary diagnosis code 430 to identify patients admitted with SAH. Patients were categorized into those admitted in border states (i.e., Arizona, California, New Mexico, and Texas) and those admitted in nonborder states (the remaining 46 states). All patients were further divided by race into Hispanic, black, white, and Asian. Data from patients without information on race were excluded from further analysis.

The study variables included in the analyses were patient age, sex, race/ethnicity, and comorbidities; the latter were obtained from comorbidity data files of the Agency for Healthcare Research and Quality and included diabetes mellitus, hypertension, congestive heart failure, renal failure, chronic lung disease, and alcohol abuse. ICD-9$\mathrm{CM}$ secondary diagnosis codes were used to identify patients with atrial fibrillation (427.3), nicotine dependence (305.1), and dyslipidemia (272.0).

ICD-9-CM secondary diagnosis codes were used to identify in-hospital complications, such as pneumonia (486, 481, 482.8, and 482.3), urinary tract infection (599.0 and 590.9), sepsis (995.91, 996.64, 038, 995.92, and 999.3), deep venous thrombosis $(451.1,451.2,451.81,451.9,453.1$, 453.2, 453.8, and 453.9), pulmonary embolism (415.1), and myocardial infarction (410). We also used ICD-9-CM primary and secondary procedure codes to estimate the percentage of in-hospital procedures, such as cerebral angiography (88.41), surgical treatment (39.51 and 39.52), endovascular treatment (39.71 and 39.79), intubation (96.04), mechanical ventilation (96.72), transfusion (99.04), tracheostomy (31.2), and gastrostomy (431.1-431.9).

The admitting hospitals were classified as urban or rural and teaching or nonteaching; teaching hospitals were those that had an American Medical Association-approved residency program or were members of the Council of Teaching Hospitals. The information in NIS about hospital size based on the number of beds was used to stratify hospitals into small, medium, and large.

We determined the length of stay (LOS) and hospital charges (amount billed for services, but not the specific amounts received in payment). Discharge status retrieved from the NIS was categorized into routine; home health care; short-term hospital; other facility, including intermediate care and skilled nursing home; or death. We categorized a routine discharge as a status of no to minimal disability and any other discharge status as moderate to severe disability as previously described. ${ }^{10}$

\section{Statistical Analysis}

SAS software (version 9.1, SAS Institute) was used to convert the NIS database data into weighted counts to generate national estimates, following the recommendations of the Healthcare Cost and Utilization Project. We performed univariate analysis and chi-square tests for categorical variables and t-tests for continuous variables to identify differences in study variables and end points between patients admitted with SAH in border or nonborder states and Hispanic versus non-Hispanic patients in both border and nonborder states.

Logistic regression models were used to identify any associations between admission for SAH in border states and the odds of in-hospital death. Additional logistic regression models included only data from those patients who survived hospitalization to examine an association between admission in border states and the odds of moderate to severe disability. The logistic regression model was adjusted for age (as a continuous variable), sex (as a categorical variable), and confounding factors (as categorical variables) that were statistically significant $(p<0.05)$ in a univariate analysis.

\section{Results}

In total, 18,368 patients were admitted with SAH in 2011, including 2310 (12.6\%) Hispanic patients; 5682 $(30.9 \%)$ of all patients were admitted in border states and $12,686(69.1 \%)$ in nonborder states.

\section{Hispanic Versus Non-Hispanic Patients}

A total of 1525 (26.8\%) Hispanic and 4157 (73.2\%) non-Hispanic SAH patients were admitted in the border states. The mean age $( \pm \mathrm{SD})$ was $61 \pm 28.3$ years and $64 \pm$ 28.4 years for Hispanic and non-Hispanic SAH patients, respectively. The patient cohort included $954(62.6 \%)$ Hispanic women and $2672(64.3 \%)$ non-Hispanic women. Most Hispanic (68.6\%) and non-Hispanic (75.8\%) patients were admitted to teaching hospitals (Table 1). 
TABLE 1. Demographics of Hispanic and non-Hispanic SAH patients in border states

\begin{tabular}{|c|c|c|c|}
\hline \multirow[b]{2}{*}{ Variable } & \multicolumn{2}{|c|}{ No. of Patients (\%) } & \multirow[b]{2}{*}{$p$ Value } \\
\hline & Hispanic (n = 1525) & Non-Hispanic $(n=4157)$ & \\
\hline \multicolumn{4}{|l|}{ Comorbidities } \\
\hline Hypertension & $1202(78.8)$ & $2857(68.7)$ & 0.010 \\
\hline Diabetes mellitus & $387(25.4)$ & $557(13.4)$ & 0.002 \\
\hline Dyslipidemia & $68(4.5)$ & $213(5.1)$ & 0.600 \\
\hline Atrial fibrillation & $66(4.3)$ & $435(10.5)$ & 0.002 \\
\hline Congestive heart failure & $142(9.3)$ & $293(7.1)$ & 0.200 \\
\hline Chronic lung disease & $137(9.0)$ & $594(14.3)$ & 0.002 \\
\hline Renal failure & $129(8.5)$ & $323(7.8)$ & 0.700 \\
\hline Alcohol abuse & $103(6.8)$ & $207(5.0)$ & 0.300 \\
\hline Nicotine dependence & $189(12.4)$ & $575(13.8)$ & 0.600 \\
\hline \multicolumn{4}{|l|}{ In-hospital complications } \\
\hline Pneumonia & $116(7.6)$ & $191(4.6)$ & 0.020 \\
\hline Deep venous thrombosis & $11(0.7)$ & $64(1.5)$ & 0.200 \\
\hline Urinary tract infection & $384(25.2)$ & $661(15.9)$ & 0.009 \\
\hline Sepsis & $119(7.8)$ & $191(4.6)$ & 0.070 \\
\hline Pulmonary embolism & $10(0.7)$ & $42(1.0)$ & 0.500 \\
\hline Myocardial infarction & $14(0.9)$ & $11(0.3)$ & 0.200 \\
\hline \multicolumn{4}{|l|}{ In-hospital procedures } \\
\hline Cerebral angiography & $734(48.1)$ & $2170(52.2)$ & 0.400 \\
\hline Gastrostomy & $145(9.5)$ & $344(8.3)$ & 0.500 \\
\hline Tracheostomy & $5(0.3)$ & $26(0.6)$ & 0.500 \\
\hline Mechanical ventilation & $242(15.9)$ & $764(18.4)$ & 0.300 \\
\hline Intubation & $311(20.4)$ & $969(23.3)$ & 0.200 \\
\hline Transfusion & $105(6.9)$ & $407(9.8)$ & 0.200 \\
\hline Endovascular coiling & $327(21.4)$ & $1014(24.4)$ & 0.400 \\
\hline Surgical clipping & $334(21.9)$ & $580(14.0)$ & 0.020 \\
\hline Mean LOS in days $( \pm \mathrm{SD})$ & $13.0 \pm 31.4$ & $11.5 \pm 26.7$ & 0.001 \\
\hline Mean hospital charge in US\$ ( \pm SD) & $219,260 \pm 652,662$ & $201,249 \pm 507,103$ & $<0.001$ \\
\hline Hospital bed size & & & 0.080 \\
\hline Small & $16(1.2)$ & $126(3.2)$ & \\
\hline Medium & $196(14.5)$ & $560(14.4)$ & \\
\hline Large & $1142(84.3)$ & $3206(82.4)$ & \\
\hline \multicolumn{4}{|l|}{ Hospital location } \\
\hline Urban & $1356(100)$ & $3838(98.6)$ & \\
\hline Rural & $0(0.0)$ & $54(1.4)$ & \\
\hline Hospital teaching status & & & 0.300 \\
\hline Nonteaching & $425(31.4)$ & $942(24.2)$ & \\
\hline Teaching & $930(68.6)$ & $2951(75.8)$ & \\
\hline \multicolumn{4}{|l|}{ Discharge disposition } \\
\hline None to min disability & $691(45.3)$ & $1775(42.7)$ & 0.400 \\
\hline Moderate to severe disability & $588(38.6)$ & $1589(38.2)$ & 0.900 \\
\hline In-hospital death & $245(16.1)$ & 793 (19.1) & 0.300 \\
\hline
\end{tabular}

Table 1 presents the demographic and clinical characteristics of the SAH patients admitted in the border states. In these states, Hispanic patients had statistically significantly higher rates of hypertension $(\mathrm{p}=0.01)$ and diabetes mellitus $(\mathrm{p}=0.002)$, and non-Hispanic patients presented with higher rates of atrial fibrillation $(p=0.002)$ and chronic lung disease $(\mathrm{p}=0.002)$. Hispanic patients in border states were statistically significantly more likely than their non-Hispanic counterparts to develop pneumonia $(\mathrm{p}=0.02)$ or urinary tract infections $(\mathrm{p}=0.009)$ and tended to have more sepsis $(p=0.07)$. More Hispanic than non-Hispanic SAH patients in the border states underwent 


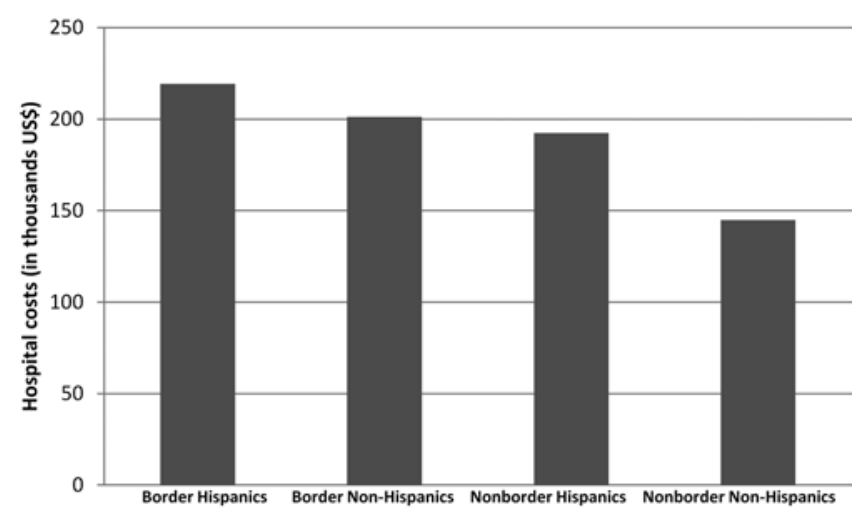

FIG. 1. Mean hospital charges for Hispanic and non-Hispanic patients with SAH in border and nonborder states. Hispanic patients admitted for SAH in border states had higher hospital charges than did their nonHispanic counterparts and Hispanic patients in nonborder states.

surgery for SAH $(21.9 \%$ vs $14.0 \%, p=0.02)$. The mean hospital LOS in the border states was $13.0 \pm 31.4$ days for Hispanic patients and $11.5 \pm 26.7$ days for non-Hispanic patients $(\mathrm{p}=0.001)$. Additionally, Hispanic patients in the border states had higher hospital charges than non-Hispanic patients ( $p<0.001)$ (Fig. 1). In border states, $16.1 \%$ of Hispanic patients admitted with SAH died in the hospital, and $19.1 \%$ of non-Hispanic patients died, but this difference was not statistically significant $(p=0.3)$. In addition, $45.3 \%$ of the Hispanic patients had none to minimal disability at discharge, and $38.6 \%$ had moderate to severe disability; $42.7 \%$ of the non-Hispanic patients had none to minimal disability at discharge, and $38.2 \%$ had moderate to severe disability. None of the differences in disability between Hispanic and non-Hispanic patients in the border states was statistically significant $(\mathrm{p}>0.05)$.

In the nonborder states, 785 (6.2\%) Hispanic patients and 11,901 (93.8\%) non-Hispanic patients were admitted for SAH (mean age $64 \pm 28$ years for both groups). Of the Hispanic patients, 497 (63.3\%) were women, and 7645 $(64.2 \%)$ of the non-Hispanic patients were women.

Table 2 presents the demographic and clinical characteristics of the SAH patients admitted in the nonborder states. Hispanic and non-Hispanic patients in these states presented similar rates of comorbidities, except for diabetes mellitus, the rate of which was again significantly higher among the Hispanic patients $(\mathrm{p}=0.01)$. In the nonborder states, Hispanic and non-Hispanic patients did not statistically significantly differ in in-hospital complications, and Hispanic patients were surgically treated for SAH at rates similar to those of non-Hispanic patients $(14.0 \%$ vs $15.6 \%, \mathrm{p}=0.6)$. The mean LOS in the nonborder states was $12.0 \pm 37.8$ days for Hispanic patients and 11.0 \pm 25.5 days for non-Hispanic patients $(\mathrm{p}<0.001)$. Similar to the findings for the border states, Hispanic patients in the nonborder states accrued higher hospital care charges than did their non-Hispanic counterparts ( $<<0.001)$. NonHispanic patients in the nonborder states tended to have higher rates of in-hospital death (23.4\%) than did Hispanic patients $(18.6 \%)(\mathrm{p}=0.07)$. In the nonborder states, $42.2 \%$ of the Hispanic patients had none to minimal disability, and $39.1 \%$ had moderate to severe disability at discharge;
$38.3 \%$ of non-Hispanic patients had none to minimal disability, and $38.3 \%$ had moderate to severe disability at hospital discharge. None of the differences in disability between Hispanic and non-Hispanic patients in the border states was statistically significant $(\mathrm{p}>0.05)$.

\section{Border Versus Nonborder States}

Table 3 shows the outcomes data for Hispanic patients in border and nonborder states. A greater proportion of patients in the nonborder states reported having dyslipidemia, that is, $10.2 \%$ of patients in nonborder states versus $4.5 \%$ of patients in border states $(p=0.03)$. Hispanic patients in border states had a higher incidence of urinary tract infections during their hospital stay (25.2\% vs $16.3 \%$, $\mathrm{p}=0.04$ ). During their stay, Hispanic patients in border and nonborder states underwent similar proportions of in-hospital procedures that included surgical clipping, endovascular coiling, cerebral angiography, intubation, and mechanical intubation. The average LOS for Hispanic patients was $13.0 \pm 31.4$ days in the border states and $12.0 \pm$ 37.8 days in the nonborder states $(\mathrm{p}=0.005)$. Hispanic patients in the border states were billed on average $\$ 219,260$ for their hospital stay, and Hispanic patients in the nonborder states had hospital costs of on average $\$ 192,418$, resulting in a statistically significant difference of $\$ 26,842$ ( $p<0.001)$. Disposition at discharge was similar for Hispanic patients in both border and nonborder states, and no statistically significant difference was detected in disability and in-hospital death between these 2 groups (Tables 4 and 5). When adjusted for age and sex, the odds ratio for in-hospital death of Hispanic patients was 0.8 (95\% CI $0.5-1.2, \mathrm{p}=0.4)$ in the border states and $0.7(95 \% \mathrm{CI}$ $0.5-1.0, p=0.09)$ in the nonborder states.

\section{Discussion}

The data of the patients with SAH in the present study were from the NIS database (collected in 2011) and were analyzed to identify any differences in outcomes and costs of treatments for SAH among Hispanic and non-Hispanic white patients in US border and nonborder states. Our analyses showed that Hispanic patients had a greater prevalence of hypertension and diabetes than non-Hispanics, regardless of state. This finding is alarming because hypertension alone increases the risk for $\mathrm{SAH} 2.5$-fold. ${ }^{1}$ Moreover, in a report from the Brain Attack Surveillance in Corpus Christi Project, conducted in a predominantly Mexican-American community, a higher prevalence of diabetes mellitus among Hispanics has been linked to a higher incidence of stroke among individuals of this group than among non-Hispanic whites. ${ }^{7}$ As the Hispanic population continues to grow, prevalence of hypertension and diabetes in this population will continue to place Hispanics at increased risk for SAH. For this reason, it is important that programs for prevention of hypertension, diabetes, and other modifiable risk factors be implemented in this population.

Our data also showed that Hispanic patients in border states were more likely than their non-Hispanic counterparts to be treated with surgical clipping. Of note, recent studies have shown that by decreasing the risk for rebleed- 
TABLE 2. Demographics of Hispanic and non-Hispanic SAH patients in nonborder states

\begin{tabular}{|c|c|c|c|}
\hline \multirow[b]{2}{*}{ Variable } & \multicolumn{2}{|c|}{ No. of Patients (\%) } & \multirow[b]{2}{*}{$p$ Value } \\
\hline & Hispanic $(n=785)$ & Non-Hispanic $(n=11,901)$ & \\
\hline \multicolumn{4}{|l|}{ Comorbidities } \\
\hline Hypertension & $616(78.5)$ & $8446(71.0)$ & 0.080 \\
\hline Diabetes mellitus & $211(26.9)$ & $2030(17.1)$ & 0.010 \\
\hline Dyslipidemia & $80(10.2)$ & $755(6.3)$ & 0.100 \\
\hline Atrial fibrillation & $50(6.4)$ & $1206(10.1)$ & 0.100 \\
\hline Congestive heart failure & $64(8.2)$ & $915(7.7)$ & 0.800 \\
\hline Chronic lung disease & $103(13.1)$ & $1733(14.6)$ & 0.600 \\
\hline Renal failure & $69(8.8)$ & $757(6.4)$ & 0.400 \\
\hline Alcohol abuse & $34(4.3)$ & $656(5.5)$ & 0.300 \\
\hline Nicotine dependence & $143(18.2)$ & $2574(21.6)$ & 0.200 \\
\hline \multicolumn{4}{|l|}{ In-hospital complications } \\
\hline Pneumonia & $45(5.7)$ & $810(6.8)$ & 0.600 \\
\hline Deep venous thrombosis & $8(1.0)$ & $214(1.8)$ & 0.300 \\
\hline Urinary tract infection & $128(16.3)$ & $1912(16.1)$ & 0.900 \\
\hline Sepsis & $42(5.4)$ & $480(4.0)$ & 0.500 \\
\hline Pulmonary embolism & $4(0.5)$ & $143(1.2)$ & 0.200 \\
\hline Myocardial infarction & $0(0.0)$ & $42(0.4)$ & \\
\hline \multicolumn{4}{|l|}{ In-hospital procedures } \\
\hline Cerebral angiography & $445(56.7)$ & $5967(50.1)$ & 0.300 \\
\hline Gastrostomy & $59(7.5)$ & $945(7.9)$ & 0.800 \\
\hline Tracheostomy & $0(0.0)$ & $27(0.2)$ & \\
\hline Mechanical ventilation & $127(16.2)$ & 1793 (15.1) & 0.700 \\
\hline Intubation & $172(21.9)$ & $2528(21.2)$ & 0.700 \\
\hline Transfusion & $64(8.2)$ & $879(7.4)$ & 0.700 \\
\hline Endovascular coiling & $236(30.1)$ & $2597(21.8)$ & 0.200 \\
\hline Surgical clipping & $110(14.0)$ & $1859(15.6)$ & 0.600 \\
\hline Mean LOS in days & $12.0 \pm 37.8$ & $11.0 \pm 25.5$ & $<0.001$ \\
\hline Mean hospital charge in US\$ ( \pm SD) & $192,418 \pm 475,784$ & $144,859 \pm 366,146$ & $<0.001$ \\
\hline Hospital bed size & & & 0.500 \\
\hline Small & $25(3.2)$ & $660(5.7)$ & \\
\hline Medium & $122(15.6)$ & $2175(18.7)$ & \\
\hline Large & $637(81.2)$ & $8796(75.6)$ & \\
\hline \multicolumn{4}{|l|}{ Hospital location } \\
\hline Urban & $785(100)$ & $11,331(97.4)$ & \\
\hline Rural & $0(0.0)$ & $300(2.6)$ & \\
\hline Hospital teaching status & & & 0.500 \\
\hline Nonteaching & $168(21.4)$ & $2989(25.7)$ & \\
\hline Teaching & $617(78.6)$ & $8643(74.3)$ & \\
\hline \multicolumn{4}{|l|}{ Discharge disposition } \\
\hline None to min disability & $331(42.2)$ & $4557(38.3)$ & 0.300 \\
\hline Moderate to severe disability & $307(39.1)$ & $4563(38.3)$ & 0.800 \\
\hline In-hospital death & $146(18.6)$ & 2781 (23.4) & 0.070 \\
\hline
\end{tabular}

ing, particularly among patients with aneurysmal SAH, endovascular coiling leads to better outcomes than surgical clipping. ${ }^{14}$ The reason why clipping was more frequently used than coiling in Hispanic patients in border states is unknown, and this lack of information may be considered a limitation of our study. Clipping compared with coiling has also been found to be associated with higher hospital charges and longer LOS of patients with both ruptured and unruptured aneurysms. ${ }^{5}$ This observation is in agreement with our findings, as Hispanic patients in the border states also had higher hospital charges and a longer LOS than their non-Hispanic counterparts and Hispanics in nonbor- 
TABLE 3. Summary of differences between Hispanic SAH patients treated in border or nonborder states

\begin{tabular}{|c|c|c|c|}
\hline \multirow[b]{2}{*}{ Variable } & \multicolumn{2}{|c|}{ No. of Patients (\%) } & \multirow[b]{2}{*}{ p Value } \\
\hline & Border State $(n=1525)$ & Nonborder State $(n=785)$ & \\
\hline Mean age in years $( \pm S D)$ & $61.0 \pm 28.3$ & $64.0 \pm 28.0$ & 0.900 \\
\hline Women & $954(62.6)$ & $497(63.3)$ & 0.800 \\
\hline \multicolumn{4}{|l|}{ Comorbidities } \\
\hline Hypertension & $1202(78.8)$ & $616(78.5)$ & 0.900 \\
\hline Diabetes mellitus & $387(25.4)$ & $211(26.9)$ & 0.700 \\
\hline Dyslipidemia & $68(4.5)$ & $80(10.2)$ & 0.030 \\
\hline Atrial fibrillation & $66(4.3)$ & $50(6.4)$ & 0.200 \\
\hline Congestive heart failure & $142(9.3)$ & $64(8.2)$ & 0.600 \\
\hline Chronic lung disease & $137(9.0)$ & $103(13.1)$ & 0.100 \\
\hline Renal failure & $129(8.5)$ & $69(8.8)$ & 0.900 \\
\hline Alcohol abuse & $103(6.8)$ & $34(4.3)$ & 0.100 \\
\hline Nicotine dependence & $189(12.4)$ & $143(18.2)$ & 0.100 \\
\hline \multicolumn{4}{|l|}{ In-hospital complications } \\
\hline Pneumonia & $116(7.6)$ & $45(5.7)$ & 0.400 \\
\hline Deep venous thrombosis & $11(0.7)$ & $8(1.0)$ & 0.600 \\
\hline Urinary tract infection & $384(25.2)$ & $128(16.3)$ & 0.040 \\
\hline Sepsis & $119(7.8)$ & $42(5.4)$ & 0.300 \\
\hline \multicolumn{4}{|l|}{ In-hospital procedure } \\
\hline Cerebral angiography & $734(48.1)$ & $445(56.7)$ & 0.300 \\
\hline Gastrostomy & $145(9.5)$ & $59(7.5)$ & 0.300 \\
\hline Mechanical ventilation & $242(15.9)$ & $127(16.2)$ & 0.900 \\
\hline Intubation & $311(20.4)$ & $172(21.9)$ & 0.600 \\
\hline Transfusion & $105(6.9)$ & $64(8.2)$ & 0.600 \\
\hline Endovascular coiling & $327(21.4)$ & $236(30.1)$ & 0.200 \\
\hline Surgical clipping & $334(21.9)$ & $110(14.0)$ & 0.100 \\
\hline Mean LOS in days $( \pm \mathrm{SD})$ & $13.0 \pm 31.4$ & $12.0 \pm 37.8$ & 0.005 \\
\hline Mean hospital charges in US\$ ( $\pm \mathrm{SD}$ ) & $219,260 \pm 652,662$ & $192,418 \pm 475,784$ & $<0.001$ \\
\hline Hospital bed size & & & 0.600 \\
\hline Small & $16(1.2)$ & $25(3.2)$ & \\
\hline Medium & $196(14.5)$ & $122(15.6)$ & \\
\hline Large & $1142(84.3)$ & $637(81.2)$ & \\
\hline \multicolumn{4}{|l|}{ Hospital location } \\
\hline Urban & $1356(100)$ & $785(100)$ & \\
\hline Rural & $0(0.0)$ & $0(0.0)$ & \\
\hline Hospital teaching status & & & 0.400 \\
\hline Nonteaching & $425(31.4)$ & $168(21.4)$ & \\
\hline Teaching & $930(68.6)$ & $617(78.6)$ & \\
\hline \multicolumn{4}{|l|}{ Discharge disposition } \\
\hline None to min disability & $691(45.3)$ & $331(42.2)$ & 0.800 \\
\hline Moderate to severe & $588(38.6)$ & $307(39.1)$ & 0.700 \\
\hline In-hospital death & $245(16.1)$ & $146(18.6)$ & 0.400 \\
\hline
\end{tabular}

der states (Fig. 1). One important factor to consider is that many Hispanics lack access to quality medical care and lack knowledge to identify the warning signs of stroke. ${ }^{8}$ Delayed treatment in this patient population may also be contributing to a longer LOS, and this delay therefore contributes to higher costs of hospitalization. Hispanics are also less likely to have health insurance, which represents another barrier to accessing needed care at the time of a stroke presentation and to stroke prevention. ${ }^{15}$ The lack of infrastructure and of trained personnel currently affecting many of the border towns may also be playing a role in why Hispanic patients in the border states are not receiving the appropriate treatment and are therefore staying longer in the hospital, resulting in additional costs. ${ }^{11}$ 
TABLE 4. Treatment outcomes among Hispanic patients in border states

\begin{tabular}{|c|c|c|c|c|c|c|}
\hline \multirow[b]{2}{*}{ Outcome } & \multicolumn{6}{|c|}{$\mathrm{OR}(95 \% \mathrm{Cl})$} \\
\hline & Unadjusted & $p$ Value & Adjusted for Age \& Sex & $\mathrm{p}$ Value & Adjusted for Age, Sex, \& Confounding Factors ${ }^{*}$ & $\mathrm{p}$ Value \\
\hline None to min disability & $1.1(0.8-1.4)$ & 0.4 & $1.0(0.8-1.3)$ & 0.8 & $1.0(0.8-1.4)$ & 0.7 \\
\hline Moderate to severe disability & $1.0(0.8-1.3)$ & 0.9 & $1.0(0.8-1.4)$ & 0.6 & $1.0(0.8-1.3)$ & 0.9 \\
\hline In-hospital death & $0.8(0.5-1.2)$ & 0.3 & $0.8(0.5-1.2)$ & 0.4 & $0.8(0.6-1.3)$ & 0.5 \\
\hline
\end{tabular}

* Only factors statistically significant (at $p<0.05$ ) in univariate analyses were included in this analysis.

TABLE 5. Treatment outcomes among Hispanic patients in nonborder states

\begin{tabular}{|c|c|c|c|c|c|c|}
\hline \multirow[b]{2}{*}{ Outcome } & \multicolumn{6}{|c|}{$\mathrm{OR}(95 \% \mathrm{Cl})$} \\
\hline & Unadjusted & $p$ Value & Adjusted for Age \& Sex & $\mathrm{p}$ Value & Adjusted for Age, Sex, \& Confounding Factors ${ }^{*}$ & $\mathrm{p}$ Value \\
\hline None to min disability & $1.2(0.8-1.6)$ & 0.30 & $1.2(0.8-1.6)$ & 0.30 & $1.2(0.8-1.6)$ & 0.30 \\
\hline Moderate to severe disability & $1.0(0.7-1.4)$ & 0.80 & $1.0(0.7-1.4)$ & 0.70 & $1.0(0.7-1.3)$ & 0.90 \\
\hline In-hospital death & $0.7(0.5-1.0)$ & 0.07 & $0.7(0.5-1.0)$ & 0.09 & $0.8(0.6-1.1)$ & 0.10 \\
\hline
\end{tabular}

* Only factors statistically significant (at $p<0.05$ ) in univariate analyses were included in this analysis.

Subarachnoid hemorrhage can be very devastating, causing $60 \%$ of SAH survivors to have some form of disability and resulting in a 50\% mortality rate; however, being white has been found to be associated with better outcomes in previous reports. ${ }^{1}$ In contrast, non-Hispanic and Hispanic patients with SAH in the border states showed similar disability and mortality rates in our study. This observation opens up the question of whether the most appropriate and cost-effective treatments are being implemented in border states.

\section{Study Limitations}

One limitation of the present study is its retrospective nature. The reporting of race in the NIS is not very accurate because race is self-reported in this database. Furthermore, the term "Hispanic" is being used for Hispanics of different descent, such as Mexican, Puerto Rican, and $\mathrm{Cu}-$ ban, whose predominance may differ among states. Also, because the NIS database was lacking a significant amount of data in specific fields, including hospital volume and zip codes of patients' residences, we were unable to characterize hospital treatment patterns or whether patients were coming to treatment sites that were close or distant to the border. Moreover, we were unable to classify patients into those who were undocumented aliens and those who were documented aliens or US citizens. Therefore, we could not perform additional analysis required for understanding the underlying reasons for the observed disparities in treatment outcomes and costs for Hispanic patients with $\mathrm{SAH}$ in border states. Another limitation was the lack of information on inclusion and exclusion criteria that were used to choose clipping versus coiling or neither treatment approach for the SAH patients in the present study.

\section{Conclusions}

Our results indicate that a disparity in treatment for SAH exists between Hispanic patients in border states and those in nonborder states. In the border states, Hispanic patients were more likely to undergo surgical clipping than non-Hispanic patients, despite the fact that coiling has been associated with better outcomes. In particular, the data in the present study also show that SAH patients in the border states were staying longer in the hospital and were being charged more per hospitalization, with Hispanic patients accruing the highest charges and having a longer LOS in the hospital. These higher charges put a greater economic burden on a population that is generally less affluent. ${ }^{15}$ Further research is needed to determine the cost-effectiveness of the SAH treatments, as mortality and disability rates were not affected by the type of treatment. Moreover, implementing interventions to prevent stroke are particularly needed for Hispanic individuals to reverse an increase in hypertension and diabetes and, in turn, avert a future rise in stroke incidence in this population.

\section{References}

1. Feigin VL, Rinkel GJ, Lawes CM, Algra A, Bennett DA, van Gijn J, et al: Risk factors for subarachnoid hemorrhage: an updated systematic review of epidemiological studies. Stroke 36:2773-2780, 2005

2. Ghaddar S, Brown CJ, Pagán JA, Díaz V: Acculturation and healthy lifestyle habits among Hispanics in United StatesMexico border communities. Rev Panam Salud Publica 28:190-197, 2010

3. Gillum RF: Albertorio-Diaz Jr, Alpert JS: Disparities in rates of acute MI hospitalization and coronary procedures on the US-Mexico border. Am J Med 123:652-630, 2010

4. Gutierrez J, Williams OA: A decade of racial and ethnic stroke disparities in the United States. Neurology 82:10801082, 2014

5. Hoh BL, Chi YY, Lawson MF, Mocco J, Barker FG II: Length of stay and total hospital charges of clipping versus coiling for ruptured and unruptured adult cerebral aneurysms in the Nationwide Inpatient Sample database 2002 to 2006. Stroke 41:337-342, 2010

6. Howard G, Goff DC: Population shifts and the future of stroke: forecasts of the future burden of stroke. Ann N Y Acad Sci 1268:14-20, 2012

7. Morgenstern LB, Smith MA, Lisabeth LD, Risser JM, Uchino K, Garcia N, et al: Excess stroke in Mexican Americans compared with non-Hispanic Whites: the Brain Attack 
Surveillance in Corpus Christi Project. Am J Epidemiol 160:376-383, 2004

8. Morgenstern LB, Steffen-Batey L, Smith MA, Moyé LA: Barriers to acute stroke therapy and stroke prevention in Mexican Americans. Stroke 32:1360-1364, 2001

9. Nasr DM, Brinjikji W, Cloft HJ, Rabinstein AA: Racial and ethnic disparities in the use of intravenous recombinant tissue plasminogen activator and outcomes for acute ischemic stroke. J Stroke Cerebrovasc Dis 22:154-160, 2013

10. Qureshi AI, Chaudhry SA, Hassan AE, Zacharatos H, Rodriguez GJ, Suri MF, et al: Thrombolytic treatment of patients with acute ischemic stroke related to underlying arterial dissection in the United States. Arch Neurol 68:1536-1542, 2011

11. Robinson KL, Ernst KC, Johnson BL, Rosales C: Health status of southern Arizona border counties: a Healthy Border 2010 midterm review. Rev Panam Salud Publica 28:344352,2010

12. Romano JG, Sacco RL: Quantifying and addressing persistent stroke disparities in Hispanics. Ann Neurol 74:759-761, 2013

13. Sacco RL, Boden-Albala B, Abel G, Lin IF, Elkind M, Hauser WA, et al: Race-ethnic disparities in the impact of stroke risk factors: the northern Manhattan stroke study. Stroke 32:1725-1731, 2001

14. van der Schaaf I, Algra A, Wermer M, Molyneux A, Clarke M, van Gijn J, et al: Endovascular coiling versus neurosurgi- cal clipping with aneurysmal subarachnoid haemorrhage. Cochrane Database Syst Rev (4):CD003085, 2005

15. Zhang X, Beckles GL, Bullard KM, Gregg EW, Albright $\mathrm{AL}$, Barker L, et al: Access to health care and undiagnosed diabetes along the United States-Mexico border. Rev Panam Salud Publica 28:182-189, 2010

\section{Disclosures}

The authors report no conflict of interest concerning the materials or methods used in this study or the findings specified in this paper.

\section{Author Contributions}

Conception and design: Hassan, Qureshi. Acquisition of data: Hassan. Analysis and interpretation of data: Hassan. Drafting the article: Hassan. Critically revising the article: Hassan, Villanueva, Malik, Shariff. Reviewed submitted version of manuscript: all authors. Statistical analysis: Hassan. Administrative/technical/ material support: Hassan. Study supervision: Hassan, Qureshi.

\section{Correspondence}

Ameer E. Hassan, Department of Neurology and Radiology, University of Texas Health Science Center-San Antonio, Valley Baptist Medical Center, 2101 Pease St., Harlingen, TX 78550. email: ameerehassan@gmail.com. 